\title{
New model for ULF Pc5 pulsations: Alfvén cones
}

\author{
P. M. Bellan \\ California Institute of Technology, Pasadena, California
}

Abstract. It is proposed that nightside auroral Pc 5 signals are inertial Alfvén resonance cones excited by localized field-aligned current pulses in the distant magnetotail.

\section{Introduction}

The nightside Pc5 pulsations often observed in auroral regions have a surprisingly reproducible, detailed structure with the following attributes: (i) Doppler radar shows [Ruohoniemi et al, 1991] that the pulsations consist of approximately east-west velocity oscillations at $\mathrm{mHz}$ frequencies [corresponding to the $\widetilde{\mathbf{E}} \times \mathbf{B}$ drift produced by a northsouth oscillatory electric field $\tilde{\mathbf{E}}_{n s}$; (ii) the spectral power at a given latitude is strongly peaked at a specific frequency which decreases monotonically with increasing latitude, and the observed frequencies appear to be quantized at $0.9,1.3$, $1.95,2.6$, and $3.3 \mathrm{mHz}$ [Ruohoniemi et al., 1991; Samson et al., 1992]; (iii) for a given observation frequency, $\overline{\mathbf{E}}_{n s}$ reverses polarity across the peak of the observed signal [Ruohoniemi et al.,1991] indicating a strong north-south shear in the east-west velocity; and (iv) although the frequency spectrum at a given latitude is nearly invariant, the time domain signal consists of a sequence of discrete wave packets [Walker et al., 1992], each at the observed frequency but with amplitude decaying after a sharp onset. The standard model for these pulsations [Hasegawa,1976; Kivelson and Southwood,1985; Samson et al.,1992] assumes they are field line resonances (FLR) excited by compressional wave cavity modes; however, Engebretson and Anderson[1995] searched satellite data and found no evidence of the assumed compressional wave cavity modes.

\section{Conceptual Basis of New Model}

We present here a new model based on the following concepts: (i) According to Goertz and Boswell [1979], Temerin et al. [1986], and Kletzing [1994], auroral pulsations are related to the inertial electron Alfven wave (IEAW). These $\omega<\omega_{c i}$ waves have the dispersion relation

$$
\dot{\omega}^{2}=k_{\|}^{2} v_{A}^{2} /\left(1+k_{\perp}^{2} c^{2} / \omega_{p e}^{2}\right),
$$

propagate when $v_{A}>\omega / k_{\|}>v_{T e}$ where $v_{T e}$ is the electron thermal velocity, and have polarization [Borg et al.,1985; Bellan,1994] $\bar{E}_{z} \neq 0, \bar{B}_{z}=0$. (ii) When excited by a localized source (i.e., continuum of $k_{\|}$, in contrast to the single, quantized $k_{\|}$of the FLR model), certain cold plasma waves have a 'resonance cone' structure [Fisher and Gould, 1969]; the IEAW is one of these waves [Kuehl, 1974; Ono, 1979; Bong et al.,1985; Bellan,1994; Gekelman et al., 1994]. For a localized source in a uniform magnetic field $\mathbf{B}=B \hat{z}$, the

\section{Copyright 1996 by the American Geophysical Union.}

Paper number 96GL01735

0094-8534/96/96GL-01735\$05.00
IEAW cone lies on the surface

$$
r / z=\omega / \omega_{g m}
$$

where $\omega_{g m}=\sqrt{\left|\omega_{c i} \dot{\omega}_{c e}\right|}=v_{A} \omega_{p e} / c$ and $r, z$ are cylindrical coordinates in a frame where the source is at the origin. Since $\omega<<\omega_{c i}<\omega_{g m}$, the IEAW cone angle is always a small fraction of a degree. (iii) If a wave has resonance cone structure, then a pulsed localized source excites a spectrum of resonance cones, each at a different angle. Superposition of these cones gives a peculiar spatial-temporal ringing pattern tern [Simonutti, 1976;Bellan, 1977]. (iv) In a non-uniform magnetic field, the cone axis follows the local field line curvature [Ohnuma, 1983] and Eq.(2) is replaced by an Eikonal relation [Bellan, 1983] which for IEAW cones will be

$$
d r / d z=\omega / \omega_{g m}(z)
$$

where $z$ is the distance from the source along the axis field line and $r$ is the radial distance from the axis field line.

\section{Derivation of the New Model}

As sketched in Fig.1 we assume that a spectrum of IEAW cones is excited by a pulse of localized field-aligned current (LFAC) associated with reconnection at a magnetotail $x$-point and that the axis of these cones is an open field line (dashed line, Fig. 1) which maps from the LFAC to a point inside the auroral oval. Each frequency component of the LFAC pulse excites a resonance cone (cf. Fig. 1) and the observed signal is the superposition of these cones. Although the cone axis is an open (lobe) field line, the cone crosses field lines as it goes earthward. Near the earth, the equatorward part of the cone can reach high-latitude closed field lines (cf. Fig. 1) while the poleward part of the cone propagates on low density open lobe field lines. We first obtain an analytic solution for a uniform plasma and then generalize to a more realistic non-uniform situation.

For the IEAW to propagate, the $v_{A}>v_{T e}$ condition must be valid along the entire propagation path. Spacecraft charging [Scime et al., 1994] typically restricts electron energy measurements to $>10 \mathrm{eV}$ making it very difficult to measure cold electrons. However, recent detailed analysis of ISEE-1 data by Sojka [private communication, 1995] has suggested the existence of a cold (i.e., under $3 \mathrm{eV}$ ) bulk electron distribution in the tail lobe at $8 R_{E}$.

We model the localized pulsed source current as $\mathbf{J}_{\text {orc }}=$ $p \hat{z} \delta(x) \delta(y) \delta(z) \delta(t)$ where $p$ is a dipole moment. Since $\omega<<$ $\omega_{c i}$, the perpendicular plasma current is the polarization current $\tilde{\mathbf{J}}_{\text {pol }}=\left(\mu_{0} v_{A}^{2}\right)^{-1} d \tilde{\mathbf{E}}_{\perp} / d t$, while the parallel plasma current (field-aligned electron motion) is given by $d \tilde{J}_{z} / d t=$ $\left(\omega_{\text {pe }}^{2} / c^{2} \mu_{0}\right) \tilde{E}_{z}$. Recalling that the IEAW has $\tilde{E}_{z} \neq 0, \tilde{B}_{z}=0$, 


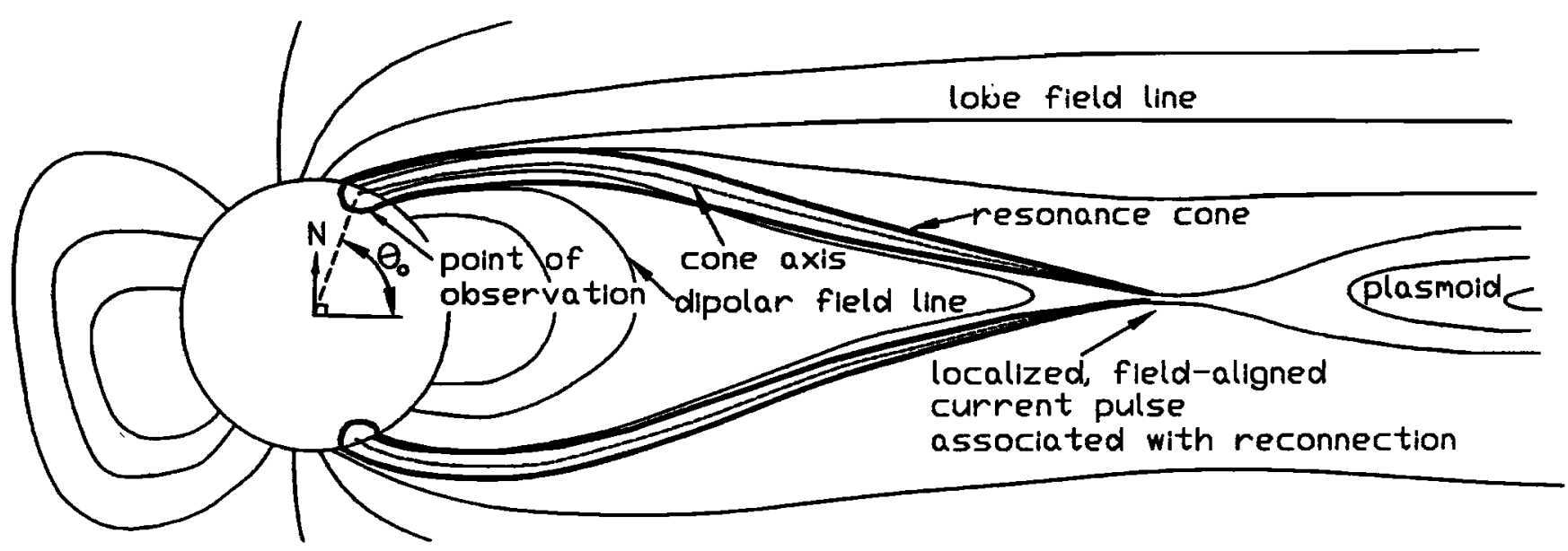

Figure 1. One cone of the continuum of IEAW resonance cones generated by a localized field-aligned current pulse. This pulse is located on an open field line in the vicinity of magnetic reconnection occurring at an $x$-point (not to scale).

the governing equations for IEAW's are the parallel and perpendicular components of Ampere's law and the perpendicular component of Faraday's law. After Laplace transforming in time and Fourier transforming in $z$, these become

$$
\begin{gathered}
\nabla_{\perp} \cdot\left(\tilde{\mathbf{B}}_{\perp} \times \hat{z}\right)=\omega_{p e}^{2} \tilde{E}_{z} / c^{2} s+\mu_{0} p \delta(x) \delta(y) \\
\hat{z} \times i k_{z} \tilde{\mathbf{B}}_{\perp}=s \tilde{\mathbf{E}}_{\perp} / v_{A}^{2}, \hat{z} \times i k_{z} \tilde{\mathbf{E}}_{\perp}+\nabla_{\perp} \tilde{E}_{z} \times \hat{z}=-s \tilde{\mathbf{B}}_{\perp}
\end{gathered}
$$

where $s$ is the Laplace transform frequency-like variable. Solving Eq.(5) for $\overline{\mathbf{E}}_{\perp}$ and $\overline{\mathbf{B}}_{\perp}$ gives

$$
\tilde{\mathbf{E}}_{\perp}=-\frac{i k_{z} v_{A}^{2}}{s^{2}+k_{z}^{2} v_{A}^{2}} \nabla_{\perp} \tilde{E}_{z}, \quad \overline{\mathbf{B}}_{\perp}=-\frac{s}{s^{2}+k_{z}^{2} v_{A}^{2}} \nabla_{\perp} \tilde{E}_{z} \times \hat{z} .
$$

Inserting $\tilde{\mathbf{B}}_{\perp}$ in Eq.(4) gives the driven IEAW equation,

$$
\nabla_{\perp} \cdot\left(\frac{s}{s^{2}+k_{z}^{2} v_{A}^{2}} \nabla_{\perp} \tilde{E}_{z}\right)-\frac{\omega_{p e}^{2}}{c^{2} s} \tilde{E}_{z}=\mu_{0} p \delta(x) \delta(y) .
$$

We now Fourier analyze in $x$ and $y$, define $k_{\perp}^{2}=k_{x}^{2}+k_{y}^{2}$ and $\xi^{2}=1+k_{\perp}^{2} c^{2} / \omega_{p e}^{2}$ so that

$$
\tilde{E}_{z}(\mathbf{k}, s)=-\frac{s \mu_{0} p\left(s^{2}+k_{z}^{2} v_{A}^{2}\right) c^{2} / \omega_{p e}^{2}}{k_{z}^{2} v_{A}^{2}+s^{2} \xi^{2}}
$$

Since the measured quantity is $\bar{E}_{x}$, it is preferable to solve for $\tilde{E}_{x}$; using Eq.(6) gives

$$
\tilde{E}_{x}(\mathbf{k}, s)=-\frac{k_{x} k_{z} s \mu_{0} p c^{2} / \omega_{p e}^{2}}{k_{z}^{2}+s^{2} \xi^{2} / v_{A}^{2}} .
$$

[Note: if we identify $s \rightarrow-i \omega$, the vanishing of the denominator in Eqs. (8) or (9) gives Eq.(1).]

Writing $k_{x}=k_{\perp} \cos \phi, d k_{x} d k_{y}=k_{\perp} d k_{\perp} d \phi$, and $r=$ $\sqrt{x^{2}+y^{2}}$, the inverse Fourier and Laplace transform of Eq.(9) at $y=0$ is

$$
\begin{array}{r}
\tilde{E}_{x}(x, z, t)=\frac{\mu_{0} p c^{2}}{i(2 \pi)^{4} \omega_{p e}^{2}} \frac{\partial^{2}}{\partial r \partial z} \int_{-i \infty}^{i \infty} d s \int_{-\infty}^{\infty} d k_{z} \int_{0}^{\infty} k_{\perp} d k_{\perp} \\
\times \int_{0}^{2 \pi} d \phi \frac{s \exp \left(i k_{\perp} r \cos \phi+i k_{z} z+s t\right)}{k_{z}^{2}+s^{2} \xi^{2} / v_{A}^{2}}
\end{array}
$$

Because of the azimuthal symmetry, $\tilde{E}_{x}(x, y=0, z, t)=$ $\tilde{E}_{r}(r, z, t)$. Integration with respect to $\phi$ gives

$$
\begin{aligned}
\tilde{E}_{r}(r, z, t)= & \frac{\mu_{0} p c^{2}}{i(2 \pi)^{3} \omega_{p e}^{2}} \frac{\partial^{2}}{\partial r \partial z} \int_{-i \infty}^{i \infty} s d s \int_{0}^{\infty} k_{\perp} d k_{\perp} \\
& \times J_{0}\left(k_{\perp} r\right) \int_{-\infty}^{\infty} d k_{z} \frac{\exp \left(i k_{z} z+s t\right)}{k_{z}^{2}+s^{2} \xi^{2} / v_{A}^{2}}
\end{aligned}
$$

where $J_{0}$ is the Bessel function of order 0 . The $k_{z}$ integrand has a simple pole in the upper half $k_{z}$-plane, so $k_{z}$ integration gives

$$
\tilde{E}_{r}=\frac{\lambda c^{2}}{\omega_{p e}^{2}} \frac{\partial^{2}}{\partial r \partial z} \int_{-i \infty}^{i \infty} d s \int_{0}^{\infty} k_{\perp} d k_{\perp} J_{0}\left(k_{\perp} r\right) \frac{e^{s t-\alpha \xi}}{\xi}
$$

where $\alpha=s z / v_{A}, \lambda=\mu_{0} p v_{A} / 8 \pi^{2}$, and the argument $(r, z, t)$ is implied for $\bar{E}_{r}$. Noting that $\xi d \xi=k_{\perp} d k_{\perp} c^{2} / \omega_{\text {pe }}^{2}$, Eq.(12) becomes

$$
\tilde{E}_{\tau}=\lambda \frac{\partial^{2}}{\partial r \partial z} \int_{-i \infty}^{i \infty} d s \int_{1}^{\infty} d \xi J_{0}\left(\frac{\omega_{p e} r}{c} \sqrt{\xi^{2}-1}\right) e^{s t-\alpha \xi}
$$

Using an integral tabulated in Gradshteyn and Ryzhik[1965], Eq.(13) becomes

$$
\tilde{E}_{r}=\lambda \frac{\partial^{2}}{\partial r \partial z} \int_{-i \infty}^{i \infty} d s \frac{\exp \left(s t-\sqrt{s^{2} z^{2} / v_{A}^{2}+\omega_{p e}^{2} r^{2} / c^{2}}\right)}{\sqrt{s^{2} z^{2} / v_{A}^{2}+\omega_{p e}^{2} r^{2} / c^{2}}} .
$$

Using an integral tabulated in Bateman [1954] this becomes,

$$
\tilde{E}_{r}(r, z, t)=\frac{i p}{4 \pi \epsilon_{0}} \frac{v_{A}^{2}}{c^{2}} \frac{\partial^{2} \Psi(r, z, t)}{\partial r \partial z}
$$

where

$$
\begin{aligned}
\Psi(r, z, t) & =0 \text { if } t<z / v_{A} \\
& =z^{-1} J_{0}\left(\frac{\omega_{g m} r}{z} \sqrt{t^{2}-z^{2} / v_{A}^{2}}\right) \text { if } t>z / v_{A}
\end{aligned}
$$

is the spatial-temporal ringing pattern, an exact solution to the $\omega<<\omega_{c i}$ Maxwell-Lorentz equations.

Equation (16) shows that the energy of the pulse extends axially from $z=0$ to $z=v_{A} t$ so that, as expected from 
causality, the wave front has a retarded time effect associated with propagation at a finite velocity. As shown in Fig.1, the poleward part of the cone propagates in low density lobe plasma which has an Alfvén velocity $v_{A}^{\text {polar }}$ that is much faster than the Alfvén velocity $v_{A}^{e q t r}$ for the equatorward part. Thus, when the poleward half of the cone reaches Earth, its axial extent will be $v_{A}^{\text {polar }} / v_{A}^{\text {eqtr }}$ longer than the axial extent of the equatorward half and so will have an amplitude $v_{A}^{\text {eqtr }} / v_{A}^{\text {polar }}$ smaller. The equatorward half of the cone, being much larger in amplitude, will therefore dominate observations, and we postulate that the radar is observing this equatorward half.

If we let $s=-i \omega$ then the integrand in Eq.(14) becomes the IEAW cone in Eq.(26) of Borg et al. [1985] and the vanishing of the denominator gives Eq.(2). Consider now how such a cone deforms when $B=B(z)$ as in Fig.1. In this case, Eq.(3) can be integrated to give

$$
r(z)=\int_{0}^{z} d z^{\prime} f / f_{g m}\left(z^{\prime}\right) ;
$$

where $f=\omega / 2 \pi, f_{g m}=\omega_{g m} / 2 \pi$. The major contribution to this integral is from the distant tail lobe where $f_{g m}(z)$ is small and the $z$-extent is large. Because the tail lobe magnetic field is very weak, non-linear wave effects in the tail lobe could, in principle, modify cone propagation. Since the plasma $\mathbf{E} \times \mathbf{B}$ motion is torsional (i.e., azimuthal) while the perpendicular phase dependence is radial, simple $\mathbf{E} \times \mathbf{B}$ convective nonlinearity seems unlikely. Other more subtle nonlinearities might occur, but for the sake of brevity, analysis of these will be deferred to future presentations.

\section{Comparison with Observations}

\section{Distance to Source}

Slavin [1985] showed that the distant tail lobe magnetic field decays as the square root of the distance from earth. Thus $f_{g m}(z)=\left(1-z / z_{s}\right)^{-1 / 2} f_{g m}^{s}$ where $z$ is the distance from the measuring point to the source, $z_{s}$ is the distance from the source to the earth and superscript $s$ means evaluated at the source. Using this $f_{g m}(z)$ in Eq.(17) and integrating from $z=0$ to $z=z_{s}$ gives the cone radius $r$ at the surface of the earth to be

$$
r=2 f z_{s} / 3 f_{g m}^{s} .
$$

Slavin also showed that the tail lobe magnetic field strength was $15 \mathrm{nT}$ at 60 earth radii so we may write $B^{s}=15 \times$ $\left(60 / z_{s}\right)^{1 / 2} \mathrm{nT}$. Since $f_{g m}=0.65 B_{n T} / \sqrt{A}$ where $A$ is mass in amu, we find $f_{g m}^{s}=76 /\left(A z_{s}\right)^{1 / 2}$. Thus Eq.(18) gives

$$
z_{s}=23[r / f]^{2 / 3} A^{-1 / 3} \text {. }
$$

If $\theta_{0}$ is the latitude of the cone axis [cf. Fig.1], the cone radius $r$ is locally related to latitude by

$$
r=2 \pi\left(\theta_{0}-\theta\right) / 360
$$

where $\theta_{0}$ is a constant. From Eq.(18) we see that $r / f$ $=$ constant; thus two frequency/latitude pairs satisfy $r_{1} / f_{1}=$ $r_{2} / f_{2}$ and so using Eq.(20), we see that $\left(\theta_{0}-\theta_{1}\right) / f_{1}=$ $\left(\theta_{0}-\theta_{2}\right) / f_{2}$. Walker et al. [1992] observed that the 3.3 $\mathrm{mHz}$ signal peaked at a latitude of $69.4^{\circ}$ while the $1.3 \mathrm{mHz}$ signal peaked at a latitude of $72.5^{\circ}$; this frequency/latitude pair gives $\theta_{0}=74.5^{\circ}$ and $r=0.089$ at $3.3 \mathrm{mHz}$. If the ion species is assumed to be mainly oxygen $(A=16)$ then Eq.(19) gives $z_{s}=82$ and $B^{s}=13 \mathrm{nT}, f_{g m}^{s}=2 \mathrm{~Hz}, f_{c i}^{s}=12$ $\mathrm{mHz}$; assuming hydrogen $(A=1)$ gives $z_{s}=206$ and $B^{s}=8$
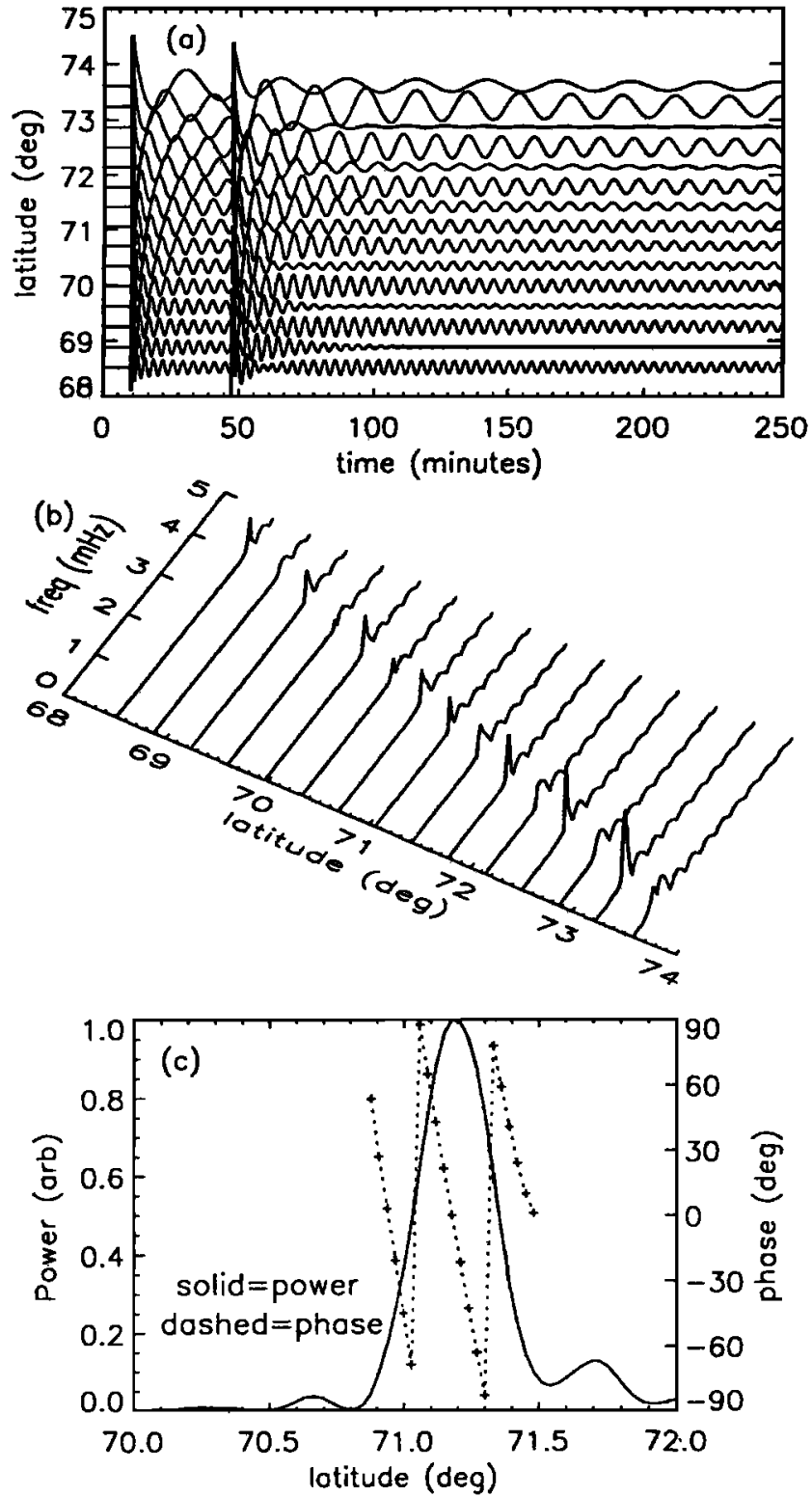

Figure 2. (a) Spatial-temporal ringing pattern $\Psi$ v. time at a sequence of latitudes; (b) spectral amplitude of $\Psi$ in (a) as a function of latitude and frequency; (c) power and phase v. latitude for $f=2.5 \mathrm{mHz}$ from FFT of $\Psi$ used in (a).

$\mathrm{nT}, f_{g m}^{s}=5 \mathrm{~Hz}, f_{c i}^{s}=120 \mathrm{mHz}$. These results are consistent with Kivelson et al.'s[1993] report of the Galileo earth encounter where a TCR-related torsional magnetic pulse in the south tail lobe at $z_{8}=\mathbf{8 0}$ appeared to be a precursor for Pc 5 signals observed 19 minutes later at Fort Churchill.

\section{Temporal, Spectral and Phase Behavior}

We now show that the pattern prescribed by $\Psi(r, z, t)$ closely resembles radar and ground station observations. Using Eq.(18), the non-uniform field version of $\Psi$ will have the argument factor $3 \omega_{g m}^{s} r\left(z_{s}\right) / 2 z_{s}$ instead of $\omega_{g m} r / z$. Figure 2(a) gives a plot of $\Psi$ v. latitude and time for two pulses separated by 37 minutes assuming an $n=10^{4} \mathrm{~m}^{-3}$ oxygen plasma with $z_{s}=82, f_{g m}^{s}=2 \mathrm{~Hz}$. These $\Psi$ plots closely resemble Figs.2 and 3 of Walker et al [1992] if we assume that Walker et al are observing the equatorward half of the cone; 
both Fig.2(a) and Walker et al.'s observations consist of a sequence of 'hard-onset' ringing oscillations, each starting at a definite time and existing over a range of latitudes such that the ringing frequency is largest at the lowest latitudes (also see Simonutti [1976]). Figure 2(b), the spectral amplitude of the Fig.2(a) signal, demonstrates that the frequency decreases with latitude in excellent agreement with Fig.1 of Samson et al.[1992]. Figure 2(c) shows spectral power and phase for one particular frequency $(2.5 \mathrm{mHz})$ plotted v. latitude. Here, the spectral power peaks at one latitude and the phase jumps $\sim 180^{\circ}$ going across the peak, in excellent agreement with Fig.2 of Samson et al.[1992].

\section{Analytic Estimate of Phase and Quantization}

The phase behavior and frequency quantization can be estimated analytically by approximating $\Psi=\exp \left(i \omega_{g m} t r / z\right)$, its long-time asymptotic limit. Thus, for a time interval $0<t<T, \Psi$ may be characterized by the Fourier time series $\Psi \sim \sum A_{n} e^{i \omega_{n} t}$ where $\omega_{n}=2 \pi n / T$. Here $A_{n} \sim$ $T^{-1} \int_{0}^{T} \Psi(r, t) e^{-i \omega_{n} t}=\xi^{-1} \sin \xi e^{i \xi}$ where $\xi=\left(\omega_{g m} r / z-\right.$ $\left.\omega_{n}\right) T / 2$. We may write $\Psi \sim \sum \Psi_{n}$ where $\Psi_{n}=A_{n} e^{i \omega_{n} t}=$ $\xi^{-1} \sin \xi e^{i\left(\omega_{n} t+\xi\right)}$. Consequently, the spectral power at frequency $\omega_{n}$ is $\left|\Psi_{n}\right|^{2} \sim \xi^{-2} \sin ^{2} \xi$ which peaks when $\omega_{n}=$ $\omega_{g m} r / z$ and drops to half-maximum at $\xi \approx \pm \pi / 2$. The phase of $\Psi_{n}$ is $\omega_{n} t+\xi$ so the phase shifts from $-\pi / 2$ to $\pi / 2$ when $\xi$ goes from $-\pi / 2$ to $+\pi / 2$. Since $r$ corresponds to latitude, $\Psi_{n}$ has peak amplitude at the latitude where $\omega_{n}=\omega_{g m} r / z$ and a $180^{\circ}$ phase shift across this peak in agreement with observations.

For multiple pulses, the frequency at a given latitude does not change because each pulse always excites the same frequency components at that latitude. For two successive pulses separated by time $\tau$, the signal will be of the form $\Psi=\sum A_{n}\left(e^{i \omega_{n} t}+e^{i \omega_{n}(t-\tau)}\right)$ so that the spectral power of the $n^{t h}$ mode will be $\left|\Psi_{n}\right|^{2}=4 \xi^{-2} \sin ^{2} \xi \cos ^{2}\left(\omega_{n} \tau / 2\right)$. The $\cos ^{2}\left(\omega_{n} \tau / 2\right)$ factor acts as a frequency-domain 'comb' filter, passing only frequencies that are integral multiples of $\tau^{-1}$. In the time-domain, this corresponds to two successive pulses either mutually reinforcing or canceling each other depending on the sign of $\cos (\omega \tau)$ as seen in Fig.2(a). The spectral power is additionally quantized by the discreteness of the measurement time T; e.g., Ruohoniemi et al's [1991] measurements were quantized in multiples of $T^{-1}=0.325$ $\mathrm{mHz}$ (using $T=3072$ seconds from Ruohoniemi et al [1991] and also Ruohoniemi, private communication[1996]). Using $\tau=37$ minutes (typical for the time domain data), the constraint that the frequency spectrum of the two-pulse signal must be in multiples of both $T^{-1}$ and of $T^{-1}$ predicts quantization at $0.9,1.3,1.95,2.6,3.3 \mathrm{mHz}$, in agreement with the observations.

Acknowledgments; Supported by USDOE and NSF.

\section{References}

Bateman, H., Tables of Integral Transforms (McGraw-Hill, NY, 1954), V.1, p. 248, formula 24.

Bellan, P. M., Plasma Ringing Associated with Pulsed Resonance Cones, Phys. Fluids, 20, 649,1977.

Bellan, P. M., Propagation of Lower Hybrid Resonance Cones in Tokamaks, Phys. Fluids, 26, 741, 1983.

Bellan, P. M., Alfven 'Resonance' Reconsidered: Exact Equations for Wave Propagation Across a Cold Inhomogeneous Plasma, Phys. Plasmas, 1, 3523, 1994.
Borg, G. G., Brennan, M. H., Cross, R. C., Giannone, L., and Donnelly, I. J., Guided Propagation of Alfven Waves in a Tonoidal Plasma, Plasma Phys. Contr. Fus., 27, 1125, 1985.

Engebretson, M. J. and Anderson, B. J., A Search for Global Mode ULF Waves in the AMPTE CCE Data Set, IUGG XXI General Assembly(1995), paper GAB41J-21.

Fisher, R. K. and Gould, R. W., Resonance Cones in the Field Pattern of a Short Antenna in an Anisotropic Plasma, Phys. Res. Lett., 22, 1093, 1969.

Gekelman, W., Leneman, D., Maggs, J., and Vincena, S., Experimental Observation of Alfvén Wave Cones, Phys. Plasmas, $1,3775,1994$.

Goertz, C. K. and Boswell, R. W., Magnetosphere-Ionosphere Coupling, J. Geophys. Res., 84, 7239, 1979.

Gradshteyn I. S. and Ryzhik I. M., Table of Integrals, Series and Products, (English translation, Academic Press, New York, 1965) Equation 6.616.2, p.710.

Hasegawa, A., Particle acceleration by MHD surface waves and formation of aurora, J. Geophys. Res., 81,5083,1976.

Kivelson, M. J. and Southwood, D. J., Resonant ULF Waves: A New Interpretation, Geophys. Res. Lett., 12, 49, 1985.

Kivelson, M. G. et al., The Galileo Earth Encounter: Magnetometer and Allied Measurements, J. Geophys. Res., 98, 11299, 1993.

Kletzing, C. A., Electron Acceleration by Kinetic Alfven Waves, J. Geophys. Res., 99, 11095, 1994.

Kuehl, H. H., Resonance Cones for Frequencies Below the Ion Cyclotron Frequency, Phys. Fluids, 17, 1636, 1974.

Ohnuma, T., Observation of Effects of Inhomogeneous Magnetic Field on Resonance Cone Fields, Jpn. J. Appl. Phys., 22,1226,1983.

Ono, M., Cold Electrostatic Ion-Cyclotron Waves and Ion-Ion Hybrid Resonances, Phys. Rev. Lett., 42, 1267 (1979).

Ruohoniemi, J. M., Greenwald, R. A., Baker, K. B., and Samson, J. C., HF Radar Observations of Pc 5 Field Line Resonances in the Midnight/Early Morning MLT Sector, J. Geophys. Res., 96, 15697, 1991.

Samson, J. C., Harrold, B. G, Ruohoniemi, J. M., Greenwald, R. A., and Walker, A. D. M., Field Line Resonances Associated with MHD Waveguides in the Magnetosphere, Geophys. Res. Lett., 19, 441, 1992.

Scime, E. E., Phillips, J. L., Bame, S. J., Effects of spacecraft potential on 3-dimensional electron measurements in the solarwind, J. Geophys. Res., 99, 14769(1994).

Simonutti, M. D., Transient Response from a Small Antenna in an Anisotropic Plasma, Phys. Fluids, 19, 608, 1976.

Slavin, J. A., Smith, E. J. Sibeck, D. G. Baker, D. N., Zwickl, R. D., and Akasofu, S.-I., An ISEE 3 Study of Average and Substorm Conditions in the Distant Magnetotail, J. Geophys. Res., 90, 10875, 1985.

Temerin, M. McFadden, J. Boehm, Carlson, C. W., and Lotko, W., Production of Flickering Aurora and Field-Aligned Electron Flux by Electromagnetic Ion Cyclotron Waves, J. Geophys. Res., 91, 51769, 1986.

Walker, A. D. M., Ruohoniemi, J. M., Baker, K. B., and Greenwald, R. A., Spatial and Temporal Behavior of ULF Pulsations Observed by the Goose Bay HF Radar, J. Geophys. Res., 97, 12187, 1992.

P. M. Bellan, MS 128-95, Caltech, Pasadena, CA 91125 (email: pbellan@cco.caltech.edu)

(received October 16,1995; revised April 18, 1996; accepted May 31, 1996.) 